Keen to keep fit and stay fit? Then avoid these injury myths and recover quickly.

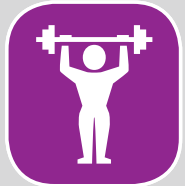

NO PAIN NO GAIN

Resting during exercise and recovery are often an overlooked part of muscle training, however it is an important factor in your exercise programme. When doing muscle training to build muscle it is important to push the strength training exercise until the point of fatigue, but not pain, before resting for a minute between sets. If you are just starting your training, begin with lighter weights that cause fatigue by the 15 th repetition. Then, as you become stronger, increase the weight to a level where you feel fatigue after 10 repetitions of the exercise.

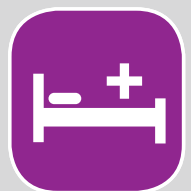

REST IS BEST

\section{AFTER SURGERY}

Following an operation, you main goal will be to regain the full movement in your joints, the full length of any muscles involved, reduce swelling and restore tissue mobility. Therefore, you will probably work with a physiotherapist who will give you exercises to complete to return strength, fitness and flexibility. However, while exercises are important, it is just as important to rest after surgery. Rest will help to control inflammation in the early stages, so it needs to be built into your overall care plan.

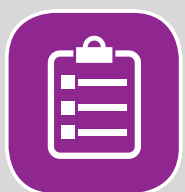

\section{REST IS BEST FOR CHRONIC (LONG TERM) PAIN}

However, if you have suffered from chronic pain over a long period, rest is not your friend. In fact, it can make your pain worse. Continued rest from long term pain leads to inflexibility, reduced strength and fitness and, over time, more pain. A graded exercise programme that gently reintroduces movement will help you break this cycle of deconditioning and help you to get your life back.

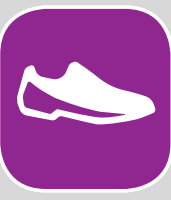

GOOD SHOES WILL CURE YOUR BACK PAIN

Footwear plays a very

important role for posture and anatomically correct movement both during sport and in our everyday lives, however while the right pair of shoes may help with back pain, it is difficult to eradicate it altogether with footwear alone. What actually works is a combination of good orthotics along with well-fitting shoes and hard work focusing on correcting your biomechanics under the guidance of your physiotherapist.

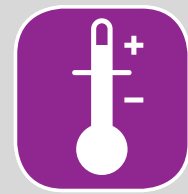

HEAT IS BETTER THAN ICE FOR PAIN

The question of whether heat or ice is better for pain has been debated for years. In truth, it is often a personal decision. Heat works better for some types of pain and ice for others. It is best to try them both to find which works best for you.

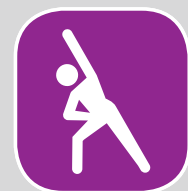

\section{STRETCH BEFORE} EXERCISE

Often people think stretching before exercise will reduce injury but there is no specific evidence to support this view. A gentle warm up involving light cardio exercise prior to exercise followed by a cool down routine will help to reduce injury.

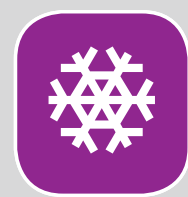

USE HEAT FOR SWELLING People often reach for a heat pad when they sustain an injury and have swelling but this will only increase blood flow to the area and increase swelling and pain. The best option is to use ice packs, cryocuffs and cold sprays.

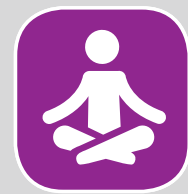

EXERCISE IS NOT GOOD FOR RHEUMATOLOGICAL CONDITIONS

Rheumatological conditions involve inflammation of the

joints and soft tissues of the body. Contrary to popular belief, exercise is beneficial to people with rheumatological conditions to improve their strength and fitness. However, the exercise programme needs to be graded and controlled to prevent any increase in inflammation or stir up of pain.

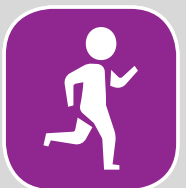

YOU CAN PREVENT RUNNING INJURIES BY JUST RUNNING

When specialising in any type of sport it is also important

to complete strengthening and stretching of the whole body to prevent injury. Alternating with different exercise programmes, for example hill runs, circuits and sprint training, will help strengthen your muscles in different ways, which can lead to faster running, increased endurance and help prevent future injury.

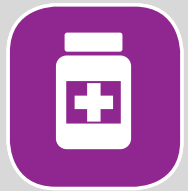

GLUCOSAMINE SULPHATE PROTECTS MY CARTILAGE FROM INJURY

There is no evidence of this and recent research has

proven no benefit of cartilage repair by taking glucosamine sulphate.

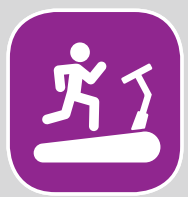

RUNNING IS HARMFUL

Running has numerous benefits, including increasing your endurance and fitness, which improves heart and

lung function. Regular running will help keep weight under control and the weight bearing part of running helps to reduce the risk of osteoporosis. Research has also shown the cartilage regeneration of joints after regular running. Pain and harm only come from having a poor running style, therefore, whether you are an experienced runner or a beginner, it is advisable to have your biomechanics checked by a physiotherapist.

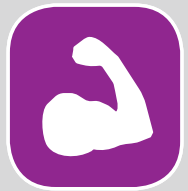

A TORN MUSCLE MEANS IT HAS BROKEN

There are different grades of tear to a muscle. A Grade 1 strain involves a small amount of damage to individual muscle fibres, causing some pain but no loss of strength. A Grade 2 strain is more extensive damage with a large number of muscle fibres involved. It causes significant pain, reduced strength and swelling. A Grade 3 strain is a complete tear of the muscle. Tears do not mean the muscle is broken and with correct advice, treatment and rehabilitation the muscle can repair and regain its strength.

bdjteam201555 